\title{
AVALIAÇÃO DE EQUINOS SUBMETIDOS À HIDRATAÇÃO ENTERAL COM SOLUÇÕES ELETROLIITICAS ISOTÔNICAS CONTENDO DIFERENTES FONTES DE ENERGIA E CÁLCIO
}

\author{
Camila Oliveira Silveira1, José Dantas Ribeiro Filho¹, Alexandra Lopes da Cruz², \\ Rodrigo Melo Meneses ${ }^{3}$, Waleska de Melo Ferreira Dantas ${ }^{1}$, José Domingos \\ Guimarães 1
}

\author{
1 UFV \\ 2 Universidade de Vila Velha \\ 3 UFMG \\ Correspondência: Camila Silveira: camilaosilveira@hotmail.com
}

\begin{abstract}
RESUMO: Avaliaram-se comparativamente os efeitos de duas soluções contendo concentrações iguais de cloreto de sódio, cloreto de potássio e magnésio, porém, uma delas contendo cloreto de cálcio e dextrose (SEDex), e a outra propionato de cálcio (SEProp), sobre parâmetros clínicos, proteínas plasmáticas totais, densidade urinária, $\mathrm{pH}$ urinário e glicose na urina de equinos (dois machos castrados e duas fêmeas) submetidos à um delineamento crossover $4 \times 2$. Os animais foram desidratados experimentalmente com duas aplicações de furosemida na dose de $1 \mathrm{mg} \mathrm{kg}^{-1}$, além de jejum hídrico-alimentar de 24 horas, anteriormente aos tratamentos com as soluções, as quais foram administradas por via enteral em fluxo contínuo através de sonda nasogástrica de pequeno diâmetro numa taxa de infusão de $15 \mathrm{mg} \mathrm{kg}^{-1}$. A desidratação induzida provocou desidratação discreta. As soluções ocasionaram expansão da volemia, diminuiu a densidade urinária e não alterou os parâmetros clínicos dos padrões de referência. Além disso, a SEProp ocasionou glicosúria.
\end{abstract}

Palavras-chave: cavalo; dextrose; hidratação; propionato de cálcio

\section{EVALUATION OF HORSES SUBJECTED TO ENTERAL FLUID THERAPY WITH ISOTONIC ELECTROLYTE SOLUTIONS CONTAINING DIFFERENT SOURCES OF ENERGY AND CALCIUM}

\begin{abstract}
This study evaluated the comparative effects of two solutions containing equal concentrations of sodium chloride, potassium chloride and magnesium, however, one containing calcium chloride and glucose (SEDex), and other calcium propionate (SEProp) on clinical parameters, total plasma protein, urine specific gravity, urinary $\mathrm{pH}$ and glucose in the urine of horses (two castrated males and two females) underwent a randomized crossover $4 \times 2$. The animals were experimentally dehydrated with two applications of furosemide at $1 \mathrm{mg} \mathrm{kg}^{-1}$, and food and water fasting for 24 hours prior to treatment with the solutions, which were administered enteral streaming through nasogastric tube of small diameter at a rate of infusion of $15 \mathrm{mg} \mathrm{kg}^{-1}$. The induced dehydration caused mild dehydration. Solutions resulted in an increase in blood volume, decreased urine specific gravity and did not alter the clinical parameters of the reference standards. Furthermore, SEProp caused glycosuria.
\end{abstract}

Key Words: calcium propionate; dextrose; horse; hydration 


\section{INTRODUÇÃO}

A desidratação ocorre devido à excessiva perda de água, com ou sem eletrólitos, juntamente à sua baixa ingestão (Swenson, 1996; Lisbôa, 2004). O tratamento consiste na hidratação do animal, que é responsável pelo restabelecimento do equilíbrio hidro-eletrolítico e ácido-base. As principais vias de administração de fluidos em equinos e bovinos correspondem às vias intravenosa e enteral, sendo a primeira a mais utilizada (Ribeiro Filho et al., 2009).

A via enteral utiliza-se de soluções não-estéreis, podendo ser utilizada água de torneira para seu preparo, uma vez que a mucosa gástrica atua como barreira seletiva de água e de eletrólitos (Ribeiro Filho et al., 2004). Em contrapartida, soluções administradas por via intravenosa devem ser estéreis e tem como principal vantagem a rápida reposição de fluidos, porém seu custo é oneroso, além de haver risco de flebite, uma vez que há a necessidade de catéter na veia durante todo o tratamento (Zimmel, 2003; DiBartola e Bateman, 2007; Avanza, 2007).

Para a administração de soluções por via enteral pode-se utilizar uma sonda nasogástrica de pequeno calibre, não impedindo que o animal se alimente enquanto recebe a hidratação, o que reduz os danos causados ao esôfago por passagens repetidas da sonda (Ribeiro Filho et al., 2004). Esse tipo de hidratação também pode aumentar 0 volume plasmático devido a uma eficiente absorção de água e eletrólitos, porém com menos rapidez quando comparado à hidratação por via intravenosa (Lopes, 2002). No entanto, em casos nos quais os pacientes apresentem distúrbios de absorção e secreção intestinal, assim como do trânsito gastrointestinal, a via enteral não deve ser utilizada (Lopes et al., 2002; DiBartola e Bateman, 2007).
A solução ideal não existe. Dessa forma, é necessário que a reidratação seja formulada de acordo com a necessidade de cada paciente, sendo imperativa, muitas vezes, a modificação da composição das soluções baseandose em exames laboratoriais e sinais clínicos para aumentar o seu valor terapêutico. Portanto, em grandes animais a hidratação por via enteral requer a elaboração de protocolos de tratamentos práticos, eficazes e baratos para a recomposição e manutenção da volemia e da homeostase desses animais (Avanza, 2007).

Algumas enfermidades determinam um quadro de inapetência ou anorexia, que pode levar à hipoglicemia. Dessa forma, torna-se necessário acrescentar às soluções eletrolíticas fontes de energia como a dextrose (Ribeiro Filho et al., 2011). O propionato de cálcio, em bovinos, fornece uma rápida fonte de cálcio absorvível, atuando também como um percussor da gliconeogênese (Fonseca et al., 1998), porém, em equinos, ainda não há estudos com essa substância. Em situações em que ocorre uma deficiência de cálcio, a administração deste elemento na solução hidratante é indicada, pois o mesmo irá atuar na estimulação peristáltica, para que ocorra a ativação das funções secretoras de contração muscular. (Thomassian, 2000; Thrall, 2007).

Objetivou-se, portanto, avaliar parâmetros clínicos e urinários e de proteínas plasmáticas totais de equinos submetidos à hidratação enteral com soluções eletrolíticas isotônicas contendo concentrações iguais de cloreto de sódio, cloreto de potássio e magnésio e diferentes fontes de cálcio e de energia administradas por via enteral em equinos desidratados experimentalmente. 

isotônicas contendo diferentes fontes de energia e cálcio

\section{MATERIAL E MÉTODOS}

Foram utilizados quatro equinos, duas fêmeas e dois machos castrados, mestiços, adultos, com bom escore corporal, peso corporal médio de $392 \mathrm{Kg}$ e clinicamente hígidos. Duas semanas antes da fase experimental, os animais foram alojados em baias individuais arejadas, com cama de serragem, limpa diariamente. Os animais receberam durante todo o período experimental feno de Tyfton 85 e água ad libitum.

Utilizou-se um protocolo de desidratação, que consistiu em jejum hídrico e alimentar de 24 horas e administração de furosemida ${ }^{1}$ por via intravenosa, na dose de $1 \mathrm{mg} \mathrm{kg}^{-1}$, duas aplicações com intervalo de 12 horas. Após a desidratação iniciou-se a hidratação dos animais por via enteral, fornecendo feno à vontade e prosseguindo com a restrição hídrica. Os animais foram submetidos, no total, a quatro períodos de 48 horas, abrangendo a desidratação, a hidratação, a observação e a coleta de amostras. Entre os períodos houve sete dias de intervalo.

As soluções foram administradas numa taxa de infusão de $15 \mathrm{~mL} \mathrm{~kg}^{-1} \mathrm{~h}^{-1}$, durante 12 horas, em fluxo contínuo, por meio de uma sonda nasogástrica ${ }^{2}$ com $4,7 \mathrm{~mm}$ de diâmetro externo e dois metros de comprimento fabricada para nutrição enteral. A sonda foi acoplada em um equipo espiral a um galão ${ }^{3} \mathrm{com}$ capacidade para 20 litros, posicionados a aproximadamente um metro de altura da cabeça de cada animal, sendo local de armazenamento das soluções durante a hidratação. A água para o preparo das soluções foi previamente aquecida em baldes até temperatura de

\footnotetext{
${ }^{1}$ LASIX

${ }^{2}$ Veterinary Enteral Feeding Tube, model: NG 145014 frenche x 50 Inch, Mila International Inc. ${ }^{\circledR}$, Florence/ Ky, EUA

${ }^{3}$ NUNES F. PLASTSOL - CNPJ 02205 335/000171
}

$37^{\circ} \mathrm{C}$ por meio de ebulidores ${ }^{4}$ e foi proveniente da estação de tratamento de água (ETA 2, na região da Violeira), da Universidade Federal de Viçosa (UFV), fornecida aos animais do Hospital Veterinário (HOV).

Utilizou-se o desenho experimental crossover $4 \times 2$, no qual os quatro animais receberam alternadamente duas soluções distintas: SEDex $(6 \mathrm{~g}$ $\mathrm{NaCl}^{5}, \quad 0,5 \mathrm{~g} \mathrm{KCl}{ }^{6}, 0,2 \mathrm{~g}$ pidolato de magnésio ${ }^{7}, 1 \mathrm{~g} \mathrm{CaCl}_{2}^{8} \mathrm{e} 1 \mathrm{~g}$ dextrose ${ }^{9} \mathrm{em}$ $1 \mathrm{~L}$ de água) e SEProp $(6 \mathrm{~g} \mathrm{NaCl}, 0,5 \mathrm{~g}$ $\mathrm{KCl}, 0,2 \mathrm{~g}$ pidolato de magnésio e $10 \mathrm{~g}$ propionato de cálcio $^{10}$ em $1 \mathrm{~L}$ água). A ordem dos tratamentos foi determinada de forma aleatória e em bloco, de acordo com o sexo dos animais conforme a tabela 1.

\begin{tabular}{|c|c|c|c|c|}
\hline Períodos & Macho 1 & Macho 2 & Fêmea 1 & Fêmea 2 \\
\hline Periodo 1 & SEDex & SEFrop & SEDex & SEFrop \\
\hline Período 2 & SEFToP & SEDEX & SEProp & SEDex \\
\hline Período 3 & SEDex & SEProp & SEDex & SEPTop \\
\hline Períod o 4 & SEPToD & SEDEx & SEProp & SEDEx \\
\hline
\end{tabular}

Os tempos de observação, exame e coleta de amostras tiveram a seguinte distribuição: T0i - imediatamente antes do início da desidratação experimental (Oh); T24i - final do período de desidratação (24h); T6t - seis horas de hidratação (6h); T12t - término do

\footnotetext{
${ }^{4}$ W RESISWAL Ebulidor 1000W/ 127V

${ }^{5}$ Química Sulfal Limitada - Cloreto de Sódio USP Rua São Rafael, 19 - Floresta - BHZ/MG, CEP: 31.015-250.

${ }^{6}$ Química Sulfal Limitada - Cloreto de Potássio USP - Rua São Rafael, 19 - Floresta - BHZ/MG, CEP:

31.015-250.

${ }^{7}$ Pidomag sol. Oral $150 \mathrm{mg} / \mathrm{mL}$ - Laboratórios Baldacci S.A. Rua Pedro de Toledo, 520, Vl. Clementino - São Paulo - SP.

${ }^{8}$ Química Sulfal Limitada - Cloreto de Cálcio Granulado - Rua São Rafael, 19 - Floresta BHZ/MG, CEP: 31.015-250.

${ }^{9}$ Dextrose $\AA$ MaxNutry $1 \mathrm{Kg}$ - Nutryclin Alimentos Ind. Brasileira - Rua José Lustosa, 63 - Bairro Silvestre - Viçosa/MG - CEP: 36.570-000.

${ }^{10}$ Calcium Propionate ${ }^{\circledR}$ FCC $25 \mathrm{Kg}$ - Kemira Chemsolutions - P.O box $60-4000$ ABTIEL Holand.
} 
período de hidratação (12h); T24f - 12 horas após o término da hidratação (correspondente à fase de avaliação final).

Dentre as variáveis de exame físico, a frequência cardíaca foi aferida através da auscultação dos batimentos cardíacos durante um minuto entre o terceiro e quinto espaço intercostal esquerdo; os movimentos respiratórios foram mensurados através da auscultação da passagem de ar na traqueia durante um minuto; a temperatura retal foi verificada por meio da utilização de um termômetro digital, em graus Celcius $\left({ }^{\circ} \mathrm{C}\right)$; o tempo de enchimento capilar (TEC) foi obtido por meio da impressão digital sobre a mucosa gengival inferior, em segundos; os movimentos intestinais foram classificados através da auscultação da região das fossas paralombares e da região ventrolaterocaudal do abdômen de acordo com a intensidade e frequência dos borborigmos em: normal, hipomotilidade e hipermotilidade. A coloração de mucosa foi verificada através da observação da mucosa oral e ocular, classificando-as em: normocorada, hipercorada e pálida, enquanto o grau de apatia foi avaliado por meio de exame físico, classificandoo em: sem apatia, apatia moderada e apatia intensa. $\mathrm{O}$ grau de desidratação foi avaliado segundo Radostists et al. (2010) em: desidratação discreta, desidratação moderada e desidratação intensa. A circunferência abdominal foi realizada por meio de fita graduada ${ }^{11}$ na região do flanco, onde pequenas áreas desta região foram tricotomizadas para demarcar o local da mensuração. Para avaliação do peso corporal, os animais foram pesados por balança para capacidade de $1000 \mathrm{Kg}^{12}$. As fezes

\footnotetext{
${ }^{11}$ Produtos Veterinários Ouro Fino LTDA. Rua Vicente Golfeto, 59 - Campos Elíseos. Ribeirão Preto - São Paulo. Tel/Fax: (16) 603-2121. CNPJ: 624.462/0001-05.

${ }^{12}$ Búffalus - Balanças do Brasil.
}

também foram analisadas de acordo com sua característica, sendo as mesmas colhidas diretamente do reto e classificadas em: ressecadas, normais, amolecidas e diarreicas.

Para a determinação das proteínas plasmáticas totais, o sangue foi colhido na veia jugular, em tubos contendo EDTA, sendo as amostras imediatamente centrifugadas para obtenção do plasma e, em seguida, analisadas através de um aparelho de refratometria modelo Clinical refratometers $n^{\circ} 140^{13}$, com escala entre 0 e 12, com variação de 0,2. Já as análises urinárias foram realizadas através da coleta de urina após micção espontânea por meio de coletores de urina, sendo aferida a densidade urinária através de um aparelho de refratômetro ${ }^{14}$, com escala entre $1000 \mathrm{e}$ 1050 , e o pH urinário e a presença de glicose na urina por meio de fita reagente ${ }^{15}$.

A análise estatística foi realizada através do programa estatístico SAEG (SAEG 9.1, UFV, 2007). Os dados quantitativos foram submetidos aos testes de Lilliefors e Bartlett para verificar a normalidade dos dados e a homocedacidade das variâncias. Posteriormente, os dados foram submetidos à análise de variância (ANOVA) e quando houve significância pelo teste $F$, as médias foram comparadas pelo teste de Tukey a $5 \%$ de probabilidade de erro. Quando os dados não se mostraram normais e sem homocedacidade, foram submetidos à análise não paramétrica $e$ as médias comparadas pelo teste de KruskalWallis ou Wilcoxom com $5 \%$ de probabilidade de erro. Além disso, para

\footnotetext{
${ }^{13}$ Fuji optical Eletronics CO. LTD, Tokyo, Japão.

${ }^{14}$ ATAGO URICON - Hand Refractometer. Cat. No.

315 - Japan.

${ }^{15}$ COMBINA 11S - Human. Gesellschaft flur

Biochemica und Diagnostica mbH. Max-Planck Ring 21, D-65205, Niesbaden - Germany.
} 

isotônicas contendo diferentes fontes de energia e cálcio

algumas variáveis de parâmetros físicos utilizou-se estatística descritiva.

\section{RESULTADOS E DISCUSSÃO}

No presente estudo, a frequência cardíaca e a temperatura retal não apresentaram diferenças entre os tratamentos nos tempos, e entre os tempos, em ambos os tratamentos $(P>0,05)$ conforme apresentado na tabela 2. Ao utilizar solução concentrada de albumina e soluções eletrolíticas contendo glicose, maltodextrina $e$ sulfato de magnésio, Belli et al. (2008) e Avanza (2007), respectivamente, encontraram resultados semelhantes ao presente ensaio. Segundo Belli et al. (2008) esse fato sinaliza a ausência de estresse excessivo nos animais. A frequência respiratória (FR) não apresentou diferença $(P>0,05)$, quando analisadas entre os tempos em ambos os tratamentos. No entanto, na avaliação entre os tratamentos houve diferença $(P<0,05)$ ao final da hidratação (T12t), porém o valor da FR permaneceu na faixa de referência para a espécie equina (Speirs, 1999), ou seja, sem significado clínico. Num estudo realizado por Avanza (2007) ocorreu diferença no decorrer do tempo em um dos tratamentos utilizados, porém o autor associou ao estresse sofrido pelos animais causado pela manipulação no período das coletas.

Observou-se aumento $(P<0,05)$ no grau de desidratação no final do período (T24i) nos animais de ambos os tratamentos (SEDex e SEProp) devido o protocolo de desidratação. Seis horas após o início da hidratação (T6t), o grau de desidratação retornou àqueles encontrados antes da desidratação (T0i), demonstrando que o período de hidratação e o volume administrado foram suficientes para sua correção. Além disso, o volume da solução eletrolítica, $15 \mathrm{~mL} \mathrm{~kg}^{-1} \mathrm{~h}^{-1}$, não ocasionou desconforto nos animais.
Apesar de não se mostrar significativo $(P>0,05), \quad o$ peso dos animais diminuíram ao final do período de desidratação (T24i), tanto no tratamento SEDex quanto no SEProp. Este fato está associado ao protocolo de desidratação utilizado, pois a associação de jejum hídrico-alimentar e a administração da furosemida determinam a perda de peso corporal (Ribeiro Filho et al., 2012). Além disso, durante a fase de hidratação (T6t e T12t) houve, aparentemente, um aumento gradual do peso corporal, possivelmente decorrente da administração de soluções eletrolíticas nos animais conforme achados de Avanza (2007), Gomes (2010) e Farias (2010).

A circunferência abdominal apresentou menores valores ao final do protocolo de desidratação (T24i), em ambos os tratamentos (SEDex e SEProp), possivelmente também em consequência do protocolo de desidratação realizado nos animais. Com a administração das soluções eletrolíticas houve aumento progressivo na circunferência abdominal, tanto no SEDex quanto no SEProp, seis (T6t) e 12 horas (T12t) após o início da hidratação $(P<0,05)$. Esse acréscimo no valor da circunferência abdominal foi ocasionado pela distensão do trato gastrointestinal devido à administração de soluções eletrolíticas como foi citado por Lopes (2002), Avanza (2007) e Gomes (2010).

O teor de água das fezes diminuiu entre o início da desidratação (T0i) e o final da mesma (T24i), em ambos os tratamentos (SEDex e SEProp). Todos os animais do tratamento SEDex apresentaram fezes ressecadas ao final da fase de desidratação (T24i), assim como a sua maioria no SEProp. Essa alteração está relacionada à desidratação dos animais, uma vez que é um fator que aumenta o ressecamento do conteúdo intestinal (Pugh e 
Thompson, 1992; Doran, 1993). Nos equinos do tratamento SEDex observouse um aumento gradual no teor de água das fezes durante a fase de hidratação, sendo observado no final da hidratação (T12t) os maiores valores $(P<0,05)$, equivalentes a fezes normais. Isto decorreu da ação do volume das soluções eletrolíticas administradas, sugerindo que o volume da solução administrada foi suficiente para normalizar o teor de água das fezes. Nos animais do tratamento SEProp constatou-se diarreia ao final da fase de hidratação $(P<0,05)$. Como nos dois tratamentos foi utilizada a mesma taxa de infusão da solução eletrolítica $(15 \mathrm{~mL}$ $\mathrm{kg}^{-1} \mathrm{~h}^{-1}$ ), possivelmente a causa da diarreia foi a composição dessa solução. O propionato de cálcio contido no tratamento SEProp pode ter modificado a osmolaridade intraluminal desencadeando um efeito osmótico, e consequentemente, um amolecimento maior das fezes, confirmando os resultados obtidos por Farias (2010).

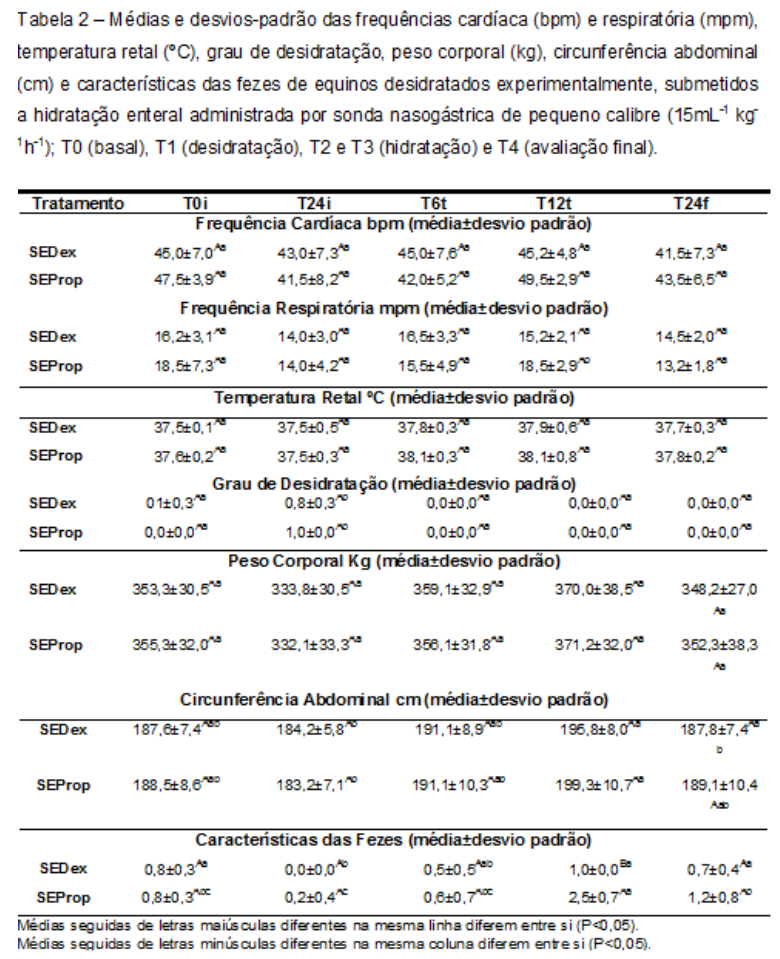

Durante a avaliação, entre os tratamentos, o tempo de enchimento capilar permaneceu dentro da faixa de referência, entre 1 e 2 segundos (Benesi e Kogika, 2002). Ao final da desidratação (T24i), em ambos os tratamentos (SEDex e SEProp), 37,5\% e $25 \%$ dos animais, respectivamente, apresentaram TEC de três segundos (tabela 3); explicada pela indução da desidratação. Alves et al. (2005) citaram que a hipovolemia induz uma baixa perfusão tecidual, limitando 0 fornecimento de oxigênio aos tecidos. Seis horas após o início da hidratação (T6t), todos os animais retornaram à faixa de normalidade, evidenciando o restabelecimento da perfusão dos tecidos.

A motilidade intestinal entre os tratamentos obteve variações, apresentando-se normal durante a fase de hidratação em $61,25 \%$ dos animais, hipomotilidade em $35 \%$ e hipermotilidade em $3,75 \%$. O tratamento SEProp demonstrou alteração de hipo ou hipermotilidade, sendo a segunda alteração a mais prevalente $(75 \%)$, possivelmente pela presença do propionato de cálcio na solução, ressaltando-se a diarreia ocorrida nos animais ao final da fase de hidratação (T12t). Em ambos os tratamentos, ao final da desidratação (T24i), a maioria dos animais apresentaram hipomotilidade (tabela 3 ). Ribeiro Filho et al. (2012) relataram que isso pode ocorrer devido ao desequilíbrio hidro-eletrolítico e ácido base ocasionados pela desidratação. Ao final da hidratação (T12t), $12,5 \%$ dos animais no tratamento SEDex e $25 \%$ no tratamento SEProp encontravam-se com hipermotilidade. Esse achado provavelmente foi ocasionado pelo estímulo do reflexo gastrocólico, como descrito por McGiness et al. (1996) e Lopes et al. (1998).

Em relação à coloração das mucosas, $97,5 \%$ dos animais apresentaram mucosas normocoradas e apenas $2,5 \%$ hipocoradas, em ambos 

isotônicas contendo diferentes fontes de energia e cálcio

os tratamentos. Imediatamente antes da hidratação, ou seja, ao final da desidratação (T24i), nos dois tratamentos (SEDex e SEProp), $12,5 \%$ dos animais apresentaram mucosas hipercoradas (tabela 3). Em casos de desidratação moderada, a mucosa se apresenta de forma pegajosa a seca e coloração avermelhada (Lisbôa, 2004). No entanto, a desidratação alcançada neste estudo foi discreta, justificando o percentual de apenas $12,5 \%$ dos equinos com essa coloração de mucosas.

Ao se avaliar o grau de apatia entre os tratamentos em cada tempo e entre os tempos em cada tratamento, $93,75 \%$ dos animais não apresentaram apatia e, apenas $6,35 \%$ dos animais do tratamento SEProp, imediatamente após o término da hidratação (T12t), demonstraram uma discreta apatia, que pode estar relacionada à quantidade de fluido que os animais receberam, uma vez que 12 horas após o término da hidratação (T24f), todos eles não se encontravam apáticos (tabela 3). Achados semelhantes foram obtidos por Ribeiro Filho (2003), porém ao final da fase de indução da compactação de cólon maior.

As proteínas plasmáticas totais aumentaram $(P<0,05)$ nos tratamentos SEDex e SEProp (Tabela 4) após a desidratação (T24i) devido ao protocolo utilizado nos animais, corroborando com achados de Alves et al. (2005) e Atoji (2005). Seis horas após o início da hidratação (T6t) e imediatamente após o final da hidratação (T12t), houve diminuição das proteínas plasmáticas, demonstrando que a hidratação enteral foi eficaz na expansão plasmática dos animais em ambos os tratamentos, fato demonstrado também por Alves et al. (2005) e Atoji (2005).

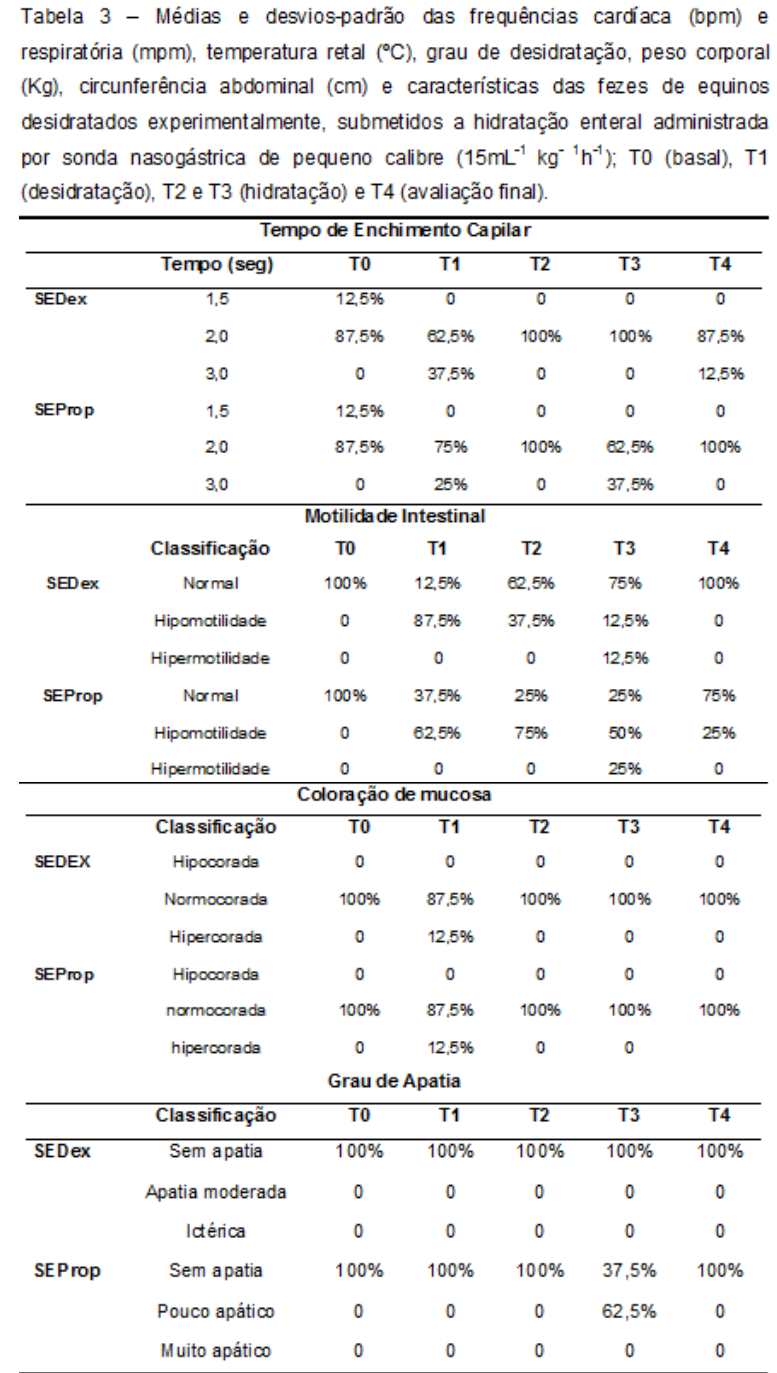

Após a desidratação dos animais (T24i), houve aumento $(P<0,05)$ da densidade urinária de ambos os tratamentos, demonstrando que 0 protocolo utilizado para desidratação foi satisfatório. Schott (1998) relata que em equinos com sistema renal normal, privados de água por 24 a 72 horas, produzem urina com densidade maior que 1045. Seis horas após a hidratação (T6t), os valores retornaram aos obtidos antes da desidratação (TOi) em ambos os tratamentos (Tabela 4). Avanza (2007) relatou que a administração de grandes quantidades de fluidos promove a diluição da urina e poliúria. Entre os tratamentos, diferença foi demonstrada ao final da hidratação (T12t), quando os animais do tratamento SEProp 
mantiveram densidade mais elevada que aqueles do tratamento SEDex. Isto pode ter ocorrido devido uma diurese osmótica provocada possivelmente pela presença de glicose na urina (Schlatter, 1997).

Não houve diferença $(P>0,05)$ no $\mathrm{pH}$ urinário entre os tempos para o tratamento SEDex, no entanto, no tratamento SEProp houve queda no $\mathrm{pH}$ da urina $(P<0,05)$ ao final da desidratação (T24i) em relação ao tempo anterior, antes da desidratação (TOi), retornando ao valor inicial seis horas após a hidratação (T6t) como pode ser visualizado na tabela 4 . Apesar dos animais do tratamento SEDex não ter apresentado redução significativa no pH urinário após a desidratação, é observado comportamento semelhante ao tratamento SEProp. A hipóxia tecidual aumenta a biossíntese de ácido láctico originário do metabolismo anaeróbico, sendo um dos principais fatores pelo aparecimento de acidose metabólica em equinos desidratados. Como a desidratação atingida foi discreta, possivelmente a excreção de íons hidrogênio $\left(\mathrm{H}^{+}\right)$pelos rins ocorreu na tentativa de manter a homeostase (Ribeiro Filho et al., 2007). Entre os tratamentos ocorreu diferença no meio da hidratação (T6t) e 12 horas após o final da mesma (T24f). No tratamento SEDex, apesar de não diferentes, percebeu-se valores menores no $\mathrm{pH}$ desde o final da desidratação até 12 horas após o término da hidratação (T24f). Isto pode estar relacionado às maiores concentrações de cloreto nessa solução eletrolítica (DiBartola e Bateman, 2007). Já no tratamento SEProp, o pH atingiu seu valor de referência após as primeiras 12 horas da administração da solução.

No tratamento SEProp, entre o T6t (seis horas após o início da hidratação) e T12t (final da hidratação) observou-se o aparecimento de glicose na urina. Isto pode ter ocorrido devido a uma quantidade excessiva de propionato de cálcio presente na solução, o qual é metabolizado no fígado, gerando glicose (Schlatter, 1997).

\begin{tabular}{|c|c|c|c|c|c|}
\hline Tratamento & TOi & T24i & T6t & T12t & T24f \\
\hline \multicolumn{6}{|c|}{ Proteinas Plasmáticas Totais $\mathrm{g} \mathrm{dl}^{-1}$ (média \pm desvio padrão) } \\
\hline SEDex & $7,0 \pm 0,4^{\varkappa}$ & $8,5 \pm 0,3^{3 s}$ & $7,1 \pm 0,4^{005}$ & $7,2 \pm 0,6^{\circ x}$ & $7,7 \pm 0,4^{40}$ \\
\hline SEProp & $7,3+0,4^{\circ}$ & $8,4 \pm 0,3^{* 0}$ & $7,5 \pm 1,3^{\infty}$ & $7,2 \pm 0,7^{\circ}$ & $7,4 \pm 0,5^{\circ \infty}$ \\
\hline \multicolumn{6}{|c|}{ Densidade Urinária (média_desvio padrão) } \\
\hline SEDex & $1016,0 \pm 7,2^{50}$ & $1030,3 \pm 8,7^{\%}$ & $1013,2 \pm 5,4^{508}$ & $1004,5 \pm 1,3^{\mathrm{cs}}$ & $1013,8 \pm 8,5^{50}$ \\
\hline SEProp & $1011,8 \pm 8,8^{\infty}$ & $1025,6 \pm 10,5^{\%}$ & $1018,2 \pm 6,3^{200}$ & $1014,2+6,5^{50}$ & $1011,4 \pm 4,1^{10}$ \\
\hline \multicolumn{6}{|c|}{ pH Urinário (média \pm desvio pa drão) } \\
\hline SEDex & $7,4 \pm 1,0^{\circ}$ & $5,9 \pm 1,0^{40}$ & $6,0 \pm 1,1^{\wedge s}$ & $6,6 \pm 0,9^{\circ}$ & $6,3 \pm 1,3^{* 0}$ \\
\hline SEProp & $7,7 \pm 0,4^{*}$ & $6,1 \pm 1,2^{5 s}$ & $7,0 \pm 0,5^{\Upsilon 00}$ & $7,5 \pm 0,7^{*}$ & $7,6 \pm 0,7^{\infty 0}$ \\
\hline \multicolumn{6}{|c|}{ Glicose (média \pm desvio padrão) } \\
\hline SEDex & $0,0 \pm 0,0$ & $0,0 \pm 0,0$ & $0,0 \pm 0,0$ & $0.0 \pm 0,0$ & $0,0 \pm 0,0$ \\
\hline SEProp & $0,0 \pm 0,0$ & $0,0 \pm 0,0$ & $0,1 \pm 0,3$ & $0,3+0,5$ & $0.0 \pm 0,0$ \\
\hline
\end{tabular}

\section{CONCLUSÃO}

O protocolo de desidratação é eficaz, enquanto a hidratação enteral por sonda nasogástrica em fluxo contínuo com soluções eletrolíticas contendo dextrose ou propionato de cálcio ocasiona a reidratação dos animais. A solução eletrolítica contendo dextrose (SEDex) determina a acidificação do pH urinário, e a que contém propionato de cálcio causa glicosúria, diarreia, desconforto abdominal e tremores musculares, tornando-a inadequada para equinos.

\section{REFERÊNCIAS}

ALVES, G.E.S; RIBEIRO FILHO, J.D.; OLIVEIRA, H.P. et al. Tratamento da compactação experimental do cólon maior em equinos: resultados de laboratório e exames bioquímicos. Arquivo Brasileiro de Medicina Veterinária e Zootecnia, v.57, n.3, p.281-287, 2005.

ATOJI, K. Fluidoterapia por via nasogástrica em caprinos. 2005. Viçosa, 98f. Dissertação (Mestrado em Medicina Veterinária) - Curso de Pós-Graduação em Medicina Veterinária, Universidade Federal de Viçosa.

AVANZA, M. F. B. Solução eletrolítica associada ou não a glicose, maltodextrina e 
Avaliação de equinos submetidos à hidratação enteral com soluções eletrolíticas isotônicas contendo diferentes fontes de energia e cálcio

sulfato de magnésio administrada por via enteral em equinos hígidos e desidratados experimentalmente. 2007. Viçosa, $100 \mathrm{f}$. Dissertação (Mestrado em Medicina Veterinária) - Curso de Pós-Graduação em Medicina Veterinária, Universidade Federal de Viçosa.

BELLI, C. B.; MICHIMA, L.E.S.; LATORRE, S.M. et. al. Solução concentrada de albumina equina na fluidoterapia em equinos com desidratação leve a moderada. Arquivo Brasileiro de Medicina Veterinária e Zootecnia, v.60, n.1, p. 30-35, 2008.

BENESI, F. J; KOGIKA, M. M. Fluidoterapia. In: SPINOSA, H. S. (Ed) Farmacologia Aplicada à Medicina Veterinária. Rio de Janeiro: Guanabara Koogan, 2002. p. 650-678.

DiBARTOLA, S. P; BATEMAN, S. Introdução à Fluidoterapia. In: DiBARTOLA, S. P (Ed)

Anormalidades de fluidos, eletrólitos e equilíbrio ácido-básico na clínica de pequenos animais. São Paulo: Roca, 2007. p. 309-328.

DORAN, R. Field management of simple intestinal obstruction in horses. Compendium on Continuing Education for the Practicing Veterinarian, v.15, n.3, p.463-471, 1993.

FARIAS, S.K.; Efeitos de soluções eletrolíticas associadas ou não à dextrose, maltodextrina e propionato de cálcio administradas por via enteral sobre parâmetros clínicos e laboratoriais de equinos, 2010. 74f. Dissertação (Master Science) - Departamento de Veterinária, Universidade Federal de Viçosa, Minas Gerais.

FONSECA, L. F.; RODRIGUES, P.H.M.; SANTOS, M.V. et. al. Supplementation of propylene glycol to dairy cows in periparturient period: effects on plasma concentration of $\mathrm{BHA}$, NEFA and glucose. Journal of Dairy Science, v.76, n.1, p.320, 1998.

GOMES, C.L.N. Efeitos do polietilenoglicol (PEG 3350) e soluções poliônicas administradas por via enteral e intravenosa em equinos. 2010. Viçosa, 130f. Tese

(Doutorado em Medicina Veterinária) - Curso de Pós-Graduação em Medicina Veterinária, Universidade Federal de Viçosa.

LISBÔA, J. A. N. Fluidoterapia em ruminantes: uma abordagem prática. In: Congresso Paulista de Medicina Veterinária, VI, 2004, Santos, Anais... Santos, 2004, v.1, p.11-15.

LOPES, M. A. F.; MOURA, G.S.; JUNQUEIRA, L.A.C. et. al. Tratamento de compactação do cólon maior em equídeos com fluidoterapia enteral. Ciência Rural, v.28, n.3, p.417-422, 1998.

LOPES, M. A. F.; WALKER, B.L.; WHITE, N.A. et. al. Treatments to promote colonic hydration: enteral fluid therapy versus intravenous fluid therapy and magnesium sulphate. Equine Veterinary Journal, v. 34, n. 5, p. 505-509, 2002.

LOPES, M. A. F. Physiological aspects, indications and contraindications of enteral fluid therapy. Equine Veterinary Education, v. 4, n.5, p.257-262, 2002.

MCGINESS, S.G.; MANSMANN, R.A.; BREUHAUS, B.A. Nasogastric electrolyte replacement in horses. Compendium on Continuing Education for the Practicing Veterinary, v.18, p. 942-950, 1996.

PUGH, D.G.; THOMPSON, J.T. Impaction colics attributed to decreased water intake and feeding coastal bermuda grass hay in a boarding stable. Equine Practice, v.14, n.1, p.9-14, 1992.

RADOSTITS, O.M.; GAY, C.C.; HINCHCLIFF, K.W. et al. Veterinary Medicine. A text book of the diseases of cattle, horses, sheep, pigs and goats. New York: Saunders, 2010, p. 2156.

RIBEIRO FILHO, J. D. Tratamento da compactação experimental do cólon maior de equinos com sene, fluidoterapia enteral e parenteral. 2003. Belo Horizonte, 130f. Tese (Doutorado em Ciência Animal) - Curso de PósGraduação em Ciência Animal, Universidade Federal de Minas Gerais.

RIBEIRO FILHO, J. D.; LOPES, M.A.F.; AVANZA, M.F.B. et. al. Tratamento de bovinos desidratados com fluidoterapia via sonda nasogástrica de pequeno calibre. In: Congresso Brasileiro de Medicina Veterinária, XXXII, 2004, São Luis, Anais... São Luis: Universitária, 2004.

RIBEIRO FILHO, J.D.; FONSECA, E.F.; MARTINS, T.M. et al. Tratamento de bovinos desidratados experimentalmente com soluções eletrolíticas por via enteral administradas por sonda nasogástrica. Archives of Veterinary Science, v. 12, n.1, p.50, 2007.

RIBEIRO FILHO, J. D.; GOMES, C.L.N.; FONSECA, B.P.A. et al. Hidratação enteral em ruminantes e equídeos. Eficiência com menor custo. Revista do Conselho Federal de Medicina Veterinária, v. 15, n.48, p.63-67, 2009.

RIBEIRO FILHO, J.D.; GIMENES, A.M.; FONSECA, E.F. et al. Hidratação enteral em bovinos: avaliação de soluções eletrolíticas isotônicas administradas por sonda nasogástrica 
em fluxo contínuo. Ciência Rural, v. 41, n. 2, p. 285-290, 2011.

RIBEIRO FILHO, J.D.; ALVES, G.E.S.;

DANTAS, W.M.F. Tratamentos da compactação experimental do cólon maior de equinos com hidratação enteral, intravenosa e sene (Cassia augustifolia Vahl). Revista Ceres, v.59, n.1, p. 407-410, 2012.

SCHLATTER, L. The addition of nutrocal to the diets of periparturient cows: a review. Des Moines: Kemin Industries, p.1-11, 1997.

SCHOTT, H. C. Examination of the urinary system. In: REED (Ed) Equine Internal Medicine. St. Louis: W. B. Saunders, 1998. p. 1200-1219.

SPEIRS, V. C. O exame clínico. In: SPEIRS (Ed) Exame Clínico de Equinos. Porto Alegre: Artes Médicas Sul Ltda, 1999.

SWENSON, M. J. Dukes - Fisiologia dos Animais Domésticos. 10 ed. Rio de Janeiro: Guanabara Koogan, 1996, 856p.

THOMASSIAN, A. Restabelecimento do trânsito intestinal em equinos - Parte I: Fisiologia e Fisiopatologia. Revista de Educação Continuada do CRMV-SP, v.3, n.1, p.14-23, 2000.

THRALL, M. A. Metabolismo de Fluidos e Eletrólitos. In: THRALL, M. A. Hematologia e Bioquímica Clínica Veterinária. São Paulo: Roca, 2007, p. 311-334.

ZIMMEL, D. N. How to manage pain and dehydration in horses with colic. In: 49th Annual Convention of the American Association of Equine Practitioners, New Orleans, Louisiana, 2003. 